\title{
Adenocarcinoma on needle prostatic biopsies: Does reactive stroma predicts biochemical recurrence in patients following radical prostatectomy?
}

Athanase Billis, Luciana Meirelles, Leandro L.L. Freitas, Aline S. Polidoro, Hamilton A. Fernandes, Mariana M. Padilha, Luis A. Magna, Leonardo 0. Reis, Ubirajara Ferreira

Department of Anatomic Pathology (AB, LM, LLLF, ASP, HAF, MMP); Medical Genetics/Biostatistics (LAM) and Urology (LOR, UF), School of Medical Sciences, State University of Campinas (Unicamp), Sao Paulo, Brazil

\section{ABSTRACT}

Objective: There is evidence that reactive stroma in different cancers may regulate tumor progression. The aim of this study is to establish any possible relation of reactive stroma grading on needle prostatic biopsies to biochemical recurrence.

Materials and Methods: The study group comprised 266 biopsies from consecutive patients submitted to radical prostatectomy. Reactive stroma was defined as stroma surrounding neoplastic tissue and graded as 0 (absent), 1 (slight), 2 (moderate), and 3 (intense) according to tumor stroma area relative to total tumor area.

Results: From the total of 266 needle prostatic biopsies, 143 (53.8\%), 55 (20.7\%), 54 (20.3\%), and 14 (5.3\%) showed grades 0, 1, 2, and 3, respectively. Increasing reactive stroma grade was significantly associated with clinical stage T2, higher preoperative PSA, higher biopsy and radical prostatectomy Gleason score, more extensive tumors in radical prostatectomy, and pathologic stage $>$ T2. Only grade 3 was significantly associated with time and risk to biochemical recurrence. On multivariate analysis only preoperative PSA and 2 methods of biopsy tumor extent evaluation were independent predictors.

Conclusion: Increasing reactive stroma grade on biopsies is significantly associated with several clinicopathologic adverse findings, however, only grade 3 predicts time and risk to biochemical recurrence following radical prostatectomy on univariate but not on multivariate analysis. We have not been able to show that reactive stroma grade 3 on biopsies is an independent predictor of biochemical recurrence beyond that of preoperative PSA and other pathologic findings on biopsy.

\section{ARTICLE INFO}

\section{Key words:}

Fibrosis; Prognosis;

Prostatic Neoplasms;

Humans; Recurrence;

Disease Progression

Int Braz J Urol. 2013; 39: 320-7

Submitted for publication:

October 08, 2012

Accepted after revision:

April 02, 2013

\section{INTRODUCTION}

Several human cancers may induce a stromal reaction (desmoplasia) as a component of carcinoma progression. This has been described in breast and colon carcinoma $(1,2)$. In cancers with stromal reaction, it seems that the response is similar, if not identical, to wound repair response
(3). Prostate cancer may also be associated with wound repair type of reactive stroma composed of myofibroblasts and fibroblasts rather than normal prostate smooth muscle, which is displaced by the reactive stroma (4).

There is growing evidence that carcinogenesis is influenced and controlled by cellular 
interactions derived from a complex relationship between stromal, epithelial and extracellular matrix components (4-15). The neoplastic stromal environment is different from the stroma of the normal tissue and is characterized by modified extracellular matrix composition, increased microvessel density, inflammatory cells and myofibroblasts $(4,14,15)$.

A pioneer study by Ayala et al. (16) showed that the volume of reactive stroma in surgical specimens from patients submitted to radical prostatectomy was a significant predictor of biochemical recurrence. In a subsequent paper from the same group, Yanagisawa et al. (17) showed that intense reactive stroma on biopsies was an independent predictor of recurrence. The only other study dealing with the prognostic value of reactive stroma in prostate carcinoma is Tomas' et al. (18). Using histochemistry and immunohistochemistry in radical prostatectomies, the authors found that on multivariate analysis only vimentin expression in reactive stroma was a significant predictor of biochemical recurrence.

Our study aims to establish the possible association of prostate cancer reactive stroma on needle biopsies and biochemical progression following radical prostatectomy.

\section{MATERIALS AND METHODS}

This retrospective study comprised 266 needle prostatic biopsies from 266 consecutive patients submitted to retropubic radical prostatectomy. No patient in this series received radiotherapy or androgen manipulation before or after surgery. The biopsy of the prostate was performed with transrectal ultrasound guidance and a spring-loaded 18-gauge needle instrument. Prostate was imaged in gray-scale mode and hypervascularity on color and power Doppler. The mean number and range of the cores obtained was 9 cores and 2-20 cores, respectively; and the mean and range of the length in $\mathrm{mm}$ of all cores 53 $\mathrm{mm}$ and 3-150 $\mathrm{mm}$, respectively. All biopsies were analyzed by the same senior uropathologist (AB).

\section{Prostatic biopsies}

The analysis was done on slides stained with hematoxylin and eosin. Reactive stroma was defined as stroma surrounding the neoplastic tissue and that was not part of the normal preexisting host stroma. The scoring system was analyzed according to the stroma area relative to total tumor area and was based on the system proposed by Ayala et al. (16) and Yanagisawa et al. (17). Reactive stroma was graded as 0 (absent or up to 5\% reactive stroma, Figures $1 \mathrm{~A}$ and B), 1 (slight, 6\% to 15\% reactive stroma, Figure-1C), 2 (moderate, $16 \%$ to $50 \%$ reactive stroma, Figure-1D), and 3 (intense, > 50\% reactive stroma, Figure-1E). In biopsies with cores showing different grades, the final grading was considered in the core with the most extensive carcinoma (index core). Reactive stroma is easily identified using hematoxylin and eosin stain and does not need special stains such as Masson's trichrome.

Grading of reactive stroma was related to several clinicopathological variables: age, clinical stage, preoperative PSA, biopsy tumor extent using several methods of evaluation, radical prostatectomy tumor extent, biopsy and radical prostatectomy Gleason score, pathologic stage, and surgical margin status. For tumor grading was used the revised Gleason system according to the consensus conference of the International Society of Urological Pathology (19).

Tumor extent on needle biopsy was evaluated as number of needle biopsy cores with carcinoma (NC), number of needle biopsy cores with carcinoma divided by the total number of cores (\%NC), total length of cancer in $\mathrm{mm}$ in all cores (mmAC), and total length of carcinoma in all cores divided by the total length of the cores (\%mmAC). Linear extent of carcinoma in mm was measured using a single micrometer eyepiece with a linear array. In cases of discontinuous foci $1 \mathrm{~mm}$ apart, the tumor was considered as continuous and the measure included $1 \mathrm{~mm}$. In discontinuous foci more than $1 \mathrm{~mm}$ apart, the final extent was the sum of the measures.

\section{Surgical specimens}

The surgical specimens were step-sectioned at 3 to $5 \mathrm{~mm}$ intervals and totally embedded in paraffin. A mean of 32 paraffin blocks were processed and $6 \mu \mathrm{m}$ sections from each block were stained with hematoxylin and eosin. Each transversal section of the prostate was subdivided into 2 anterolateral and 2 posterolateral quadrants. The basal and the apical margins were amputated and 
Figure 1 - (A) Grade 0 (absent reactive stroma): Gleason score 6 adenocarcinoma; (B) Grade 0 (absent reactive stroma): Gleason score 7 adenocarcinoma; (C) Grade 1 (slight reactive stroma); (D) Grade 2 (moderate reactive stroma); (E) Grade 3 (intense reactive stroma). Only some few residual smooth muscle fibers are seen (arrow) (hematoxylin-eosin, x165).

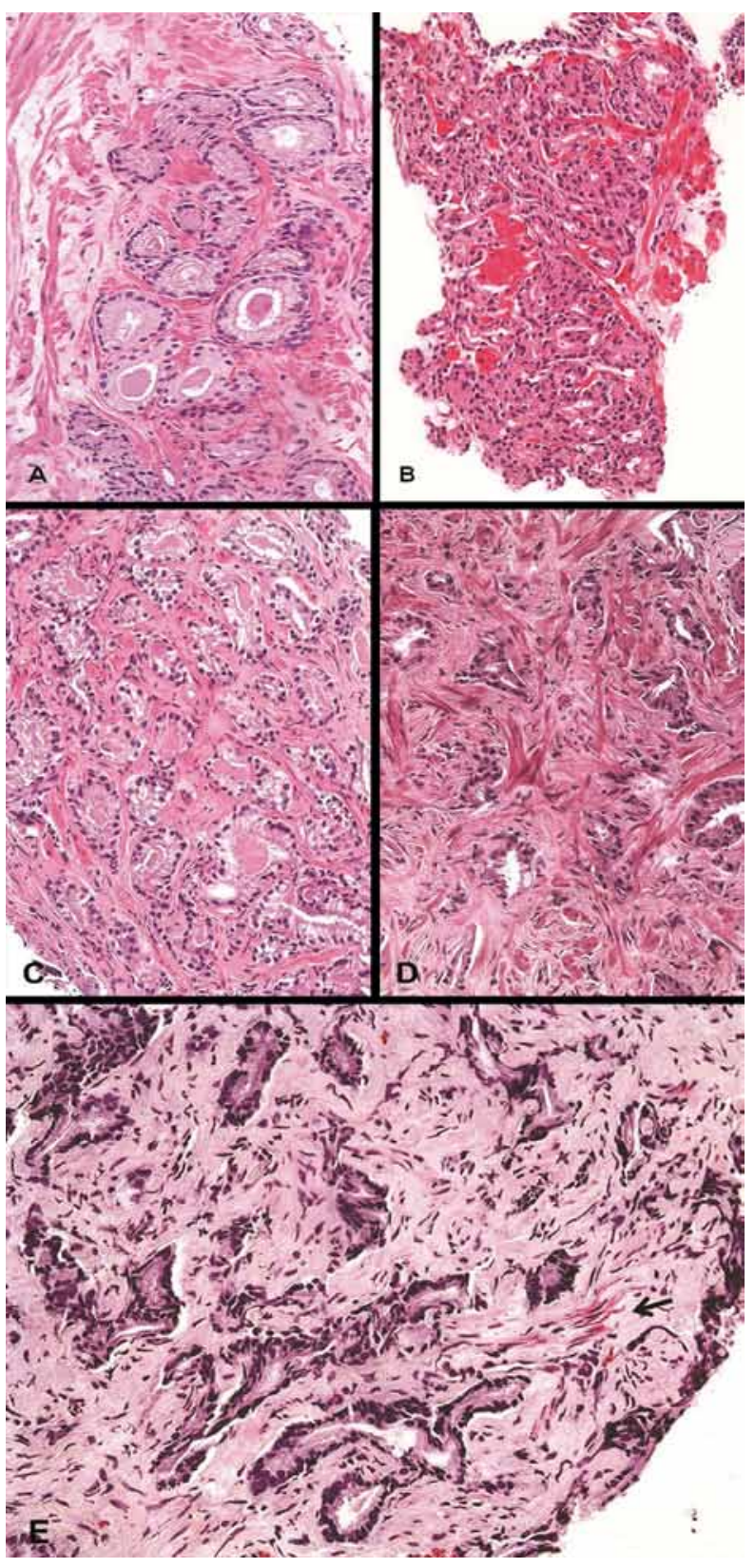

sectioned parasagittally in a direction perpendicular to the initial transverse incision (cone method) obtaining 8 sections from each margin. Positive surgical margin was defined as cancer cells in contact with the inked specimen surface. Extraprostatic extension was diagnosed whenever cancer was seen in adipose tissue and, in case of desmoplastic response, whenever a protuberance corresponding to extension of tumor into periprostatic tissue was observed. Seminal vesicle invasion occurred whenever there was involvement of the muscular coat. Tumor extent at radical prostatectomy was evaluated by a semiquantitative point-count method previously described (20).

After radical prostatectomy, serum PSA was drawn every 3 months during the first year, every 6 months during the second year, and annually thereafter. No patient had radiotherapy or androgen manipulation before or after surgery. Total serum PSA was measured utilizing previous validated Immulite ${ }^{\circledR}$ PSA kit. Biochemical recurrence following surgery was considered as PSA $\geq 0.2 \mathrm{ng} / \mathrm{mL}$ according to recommendation of the American Urological Association (21). Patients without evidence of biochemical recurrence were censored at last follow-up. Institutional Committee of Ethics approved the study.

\section{Statistical analysis}

The data were analyzed using the Qui-square test for comparison of proportions, the Kruskal-Wallis test for comparison of means, the Kaplan-Meier product-limit analysis for the time to biochemical recurrence using the log-rank test for comparison between the groups, and a univariate and multivariate Cox stepwise logistic regression model to identify significant predictors of shorter time to biochemical recurrence. The P-values were two-sided at the significance level of $<0.05$. All statistical analyses were performed using the commercially available PASW Statistics (SPSS) 18.0.

\section{RESULTS}

\section{Biopsies}

Table- 1 shows the clinicopathologic characteristics of 266 patients undergoing radical prostatectomy according to biopsy reactive stroma grade. From the total of 266 needle prostatic biopsies, 143 (53.8\%), 55 (20.7\%), 54 (20.3\%), and 14 (5.3\%) biopsies showed absent (grade 0), slight (grade 1), moderate (grade 2), and intense (grade 3) reactive stroma, respectively. 
Table 1 - Clinicopathologic characteristics of 266 patients undergoing radical prostatectomy according to biopsy reactive stroma grade.

\begin{tabular}{|c|c|c|c|c|c|}
\hline Characteristic & Grade 0 & Grade 1 & Grade 2 & Grade 3 & $P$ value \\
\hline No. of patients & $143(53.8 \%)$ & $55(20.7 \%)$ & $54(20.3 \%)$ & $14(5.3 \%)$ & \\
\hline \multicolumn{6}{|l|}{ Mean (SD; range) } \\
\hline Age, years & $64(6.6 ; 46-76)$ & $62(6.8 ; 45-73)$ & $63(7.2 ; 43-73)$ & $65(4.3 ; 57-72)$ & 0.69 \\
\hline Preoperative PSA (ng/mL) & $8.6(5 ; 0.6-29.7)$ & $9.5(4.3 ; 2.6-22.1)$ & $11(7 ; 0.9-29-7)$ & $18.1(14.7 ; 3.43-50)$ & $<0.01$ \\
\hline $\begin{array}{l}\text { RP tumor extent } \\
\text { (pos.points) }\end{array}$ & $31.4(30.9 ; 1-192)$ & $37.4(30.4 ; 1-147)$ & $46.9(45.3 ; 5-222)$ & $68.8(71.2 ; 20-225)$ & 0.02 \\
\hline \multicolumn{6}{|l|}{ N (\%) } \\
\hline \multicolumn{6}{|l|}{ Clinical stage } \\
\hline T1c & $81(58.7)$ & $23(44.2)$ & $9(19.6)$ & $2(16.7)$ & $<0.01$ \\
\hline T2 & $57(41.3)$ & $29(55.8)$ & $37(80.4)$ & $10(83.3)$ & $<0.01$ \\
\hline \multicolumn{6}{|l|}{ RP Gleason score } \\
\hline $2-6$ & $65(45.5)$ & $15(27.3)$ & $10(19.2)$ & $1(7.1)$ & \\
\hline 7 & $73(51.0)$ & $35(63.6)$ & $40(76.9)$ & $11(78.6)$ & \\
\hline $8-10$ & $5(3.5)$ & $5(9.1)$ & $2(3.8)$ & $2(14.3)$ & \\
\hline \multicolumn{6}{|l|}{ Biopsy Gleason score } \\
\hline $2-6$ & $103(72.0)$ & $30(54.5)$ & $32(59.3)$ & $7(50.0)$ & 0.02 \\
\hline 7 & $36(25.2)$ & $21(38.2)$ & $19(35.2)$ & $4(28.6)$ & \\
\hline $8-10$ & $4(2.8)$ & $4(7.3)$ & $3(5.6)$ & $3(21.4)$ & \\
\hline \multicolumn{6}{|l|}{ Pathologic stage } \\
\hline T2 & $110(76.9)$ & $42(76.4)$ & $35(64.8)$ & $5(35.7)$ & 0.01 \\
\hline > T2 (T3a/T3b) & $33(23.1)$ & $13(23.6)$ & $19(35.2)$ & $9(64.3)$ & \\
\hline \multicolumn{6}{|l|}{ Surgical margin status } \\
\hline Negative & $86(60.1)$ & $29(52.7)$ & $28(51.9)$ & $5(35.7)$ & 0.27 \\
\hline Positive & $57(39.9)$ & $26(47.3)$ & $26(48.1)$ & $9(64.3)$ & \\
\hline
\end{tabular}


Increasing reactive stroma grade was significantly associated with clinical stage T2, higher preoperative PSA, higher biopsy and radical prostatectomy Gleason score, more extensive tumors in radical prostatectomy, and pathologic stage $>\mathrm{T} 2$. There was no significant association with age and positive surgical margin.

\section{Radical prostatectomies}

From the total of 266 men following radical prostatectomy, $92(34.6 \%)$ patients had biochemical recurrence at a mean, median and range follow-up of 19, 8, and 3-111 months; 162 (60.9\%) censored men remained at risk at a mean, median and range follow-up of 59, 57, and 3-141 months, respectively; and, 12 (4.5\%) men had no serum PSA data.

At 5 years following radical prostatectomy, $69 \%, 59 \%, 57 \%$, and 39\% from a total of 254 patients with grade $0,1,2$, and 3 biopsy reactive stroma, respectively, were free of biochemical recurrence; 12/266 (4.5\%) men had no serum PSA data. Only patients with needle biopsies showing grade 3 reactive stroma were significantly associated with shorter time to biochemical recurrence following surgery (log-rank, $p<0.01$ ) (Figure-2).

Figure 2 - Kaplan-Meyer product-limit analysis comparing grades $0,1,2$, and 3 reactive stroma on needle biopsy from 254 patients for time to biochemical recurrence following radical prostatectomy.

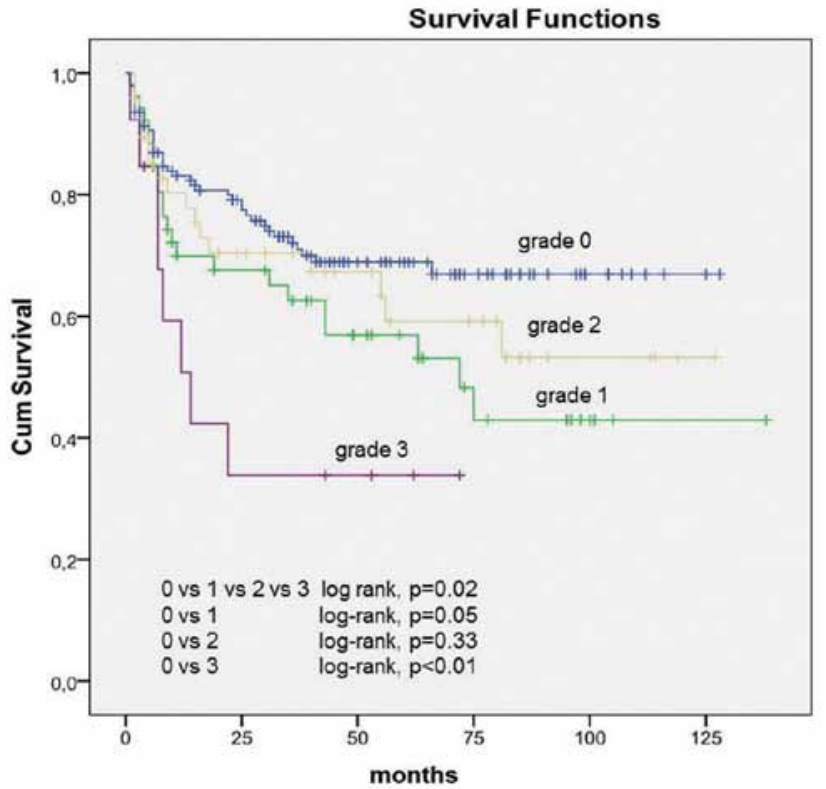

Table-2 shows the Cox proportional hazard analysis of several clinicopathologic factors predicting time to biochemical recurrence following radical prostatectomy. On univariate analysis, preoperative PSA, all methods of biopsy tumor extent evaluation, and only needle biopsies with grade 3 reactive stroma were significant predictors of time to biochemical recurrence. On multivariate analysis, only preoperative PSA and 2 methods of biopsy tumor extent evaluation were independent predictors using two models of analysis.

\section{DISCUSSION}

We used the system proposed by Ayala et al. (16) and Yanagisawa et al. (17) for definition and scoring of reactive stroma. Reactive stroma was evaluated as stroma surrounding the neoplastic tissue and not part of the normal preexisting host stroma. The scoring system was established according to the stroma area relative to total tumor area. In biopsies with cores showing different grades, the final grading was considered in the core with the most extensive carcinoma (index core). Reactive stroma is easily identified using hematoxylin and eosin stain and does not need special stains such as Masson's trichrome. No patient in this series received radiotherapy or androgen manipulation before or after surgery. This is important because both occurrences promote mesanchymal reaction that may interfere with the results.

All patients in our study had only one needle prostatic biopsy preceding surgery therefore there was no selection of biopsies. The mean number and range of the cores examined was 9 and $2-20$, respectively and the mean and range of the length in $\mathrm{mm}$ of all cores $53 \mathrm{~mm}$ and $3-150 \mathrm{~mm}$, respectively. Sampling of the biopsies is important for comparison of results.

According to intensity of reactive stroma, the frequency was 143/266 (53.8\%), 55/266 (20.7\%), $54(20.3 \%)$, and 14/266 (5.3\%) biopsies for grades $0,1,2$, and 3, respectively. The frequency is higher in grade 0 and decreases to grade 3. On needle biopsies, Yanagisawa et al. (17) found a frequency of $1 / 224(0.5 \%), 149 / 224$ (66.5\%), $59 / 224(26.3 \%)$, and 15/224 (6.7\%) biopsies for grades $0,1,2$, and 3 , respectively. In radical pros- 
Table 2 - Cox proportional hazard analysis of several clinicopathologic factors predicting time to biochemical recurrence following radical prostatectomy.

\begin{tabular}{|c|c|c|c|}
\hline Predictors & Hazard ratio $(95 \% \mathrm{Cl})$ & Wald test & $P$ value \\
\hline \multicolumn{4}{|l|}{ Univariate analysis } \\
\hline Reactive stroma grade 1 & $1.655(0.990-2.765)$ & 3.696 & 0.06 \\
\hline Reactive stroma grade 2 & $1.320(0.748-2.329)$ & 0.915 & 0.34 \\
\hline Reactive stroma grade 3 & $2.863(1.386-5.914)$ & 8.071 & $<0.01$ \\
\hline Preoperative PSA & $1.058(1.036-1.080)$ & 28.700 & $<0.01$ \\
\hline Biopsy Gleason score & $1.293(0.993-1.684)$ & 3.645 & 0.06 \\
\hline Biopsy NC & $1.126(1.038-1.221)$ & 8.139 & $<0.01$ \\
\hline Biopsy \%NC & $1.012(1.005-1.019)$ & 10.284 & $<0.01$ \\
\hline Biopsy mmAC & $1.025(1.007-1.043)$ & 7.721 & 0.01 \\
\hline Biopsy \%mmAC & $1.017(1.008-1.026)$ & 13.306 & $<0.01$ \\
\hline \multicolumn{4}{|l|}{ Multivariate analysis } \\
\hline \multicolumn{4}{|l|}{ Model 1} \\
\hline Preoperative PSA & $1.042(1.012-1.073)$ & 7.436 & 0.01 \\
\hline Biopsy \%NC & $1.011(1.001-1.001)$ & 4.288 & 0.04 \\
\hline \multicolumn{4}{|l|}{ Model 2} \\
\hline Preoperative PSA & $1.035(1.003-1.068)$ & 4.522 & 0.03 \\
\hline Biopsy \%mmAC & $1.016(1.003-1.028)$ & 6.216 & 0.01 \\
\hline
\end{tabular}

tatectomies, Ayala et al. (16) found a frequency of $34 / 545$ (6.2\%), 161/545 (29.5\%), 306/545 (56.1\%) , and 44/545 (8\%) surgical specimens for grades 0 , 1,2 , and 3 , respectively.

The higher frequency of grade $0(53.8 \%)$ in our study contrasts with the frequency of $0.5 \%$ and $6.2 \%$ of grade 0 in Yanagisawa's et al. (17) and Ayala's et al. (16) studies. Considering that the method of evaluation of reactive stroma is the same, it is difficult to explain this striking difference in frequency. Absence of reactive stroma is easily discernible as we can see in Figures $1 \mathrm{~A}$ and B. One possible reason may be the additional use of trichrome staining to visualize reactive stroma in those prior studies. There is also a role of inte- robserver variability in interpretation of reactive stroma particularly when it is absent or lower grade. However, since distinction is more important for grade 3, which is relatively easier to identify, variation of interpretation in lower grades may not be significant.

Increasing reactive stroma grade was significantly associated with adverse clinicopathologic findings. Table- 1 clearly shows the significant association with clinical stage T2, higher preoperative PSA, higher tumor extent in surgical specimen, higher Gleason score in biopsy and surgical specimen, and pathological stage $>\mathrm{T} 2$. There was no significant association with age and positive surgical margin. In Yanagisawa's et al. (17) study, 
reactive stroma grade in biopsies was significantly associated with clinical and pathological stage, surgical margin stage, and Gleason score on biopsy. No correlation was found with preoperative PSA and age.

The Kaplan-Meier product-limit analysis showed that only patients with reactive stroma grade 3 had significantly shorter time to biochemical recurrence (Figure-1). In Cox univariate analysis reactive stroma grade 3 is a significant predictor of shorter time to biochemical recurrence (Table-2). Yanagisawa et al. (17) found that patients with reactive stroma grade 0 and 3 had significantly shorter time to biochemical recurrence. A comment on this finding is the fact that they included in the same group 15 patients with reactive stroma grade 3 and only one patient with grade 0 in their series. It is not plausible any influence of this single patient with reactive stroma grade 0 in the analysis.

On multivariate analysis we used two models including only variables significantly predictive of time to biochemical recurrence on univariate analysis (Table-2). In model 1, only preoperative PSA and number of needle biopsy cores with carcinoma divided by the total number of cores (\%NC) were significant; in model 2, only preoperative PSA and the total length of carcino$\mathrm{ma}$ in $\mathrm{mm}$ in all cores divided by the total length of the cores (\%mmAC). This result is at odds with Yanagisawa's et al. (17) study on biopsies. According to the authors reactive stroma grade 0 and 3 was an independent predictor of biochemical recurrence by Cox proportional hazard analysis. Their analyses were based on 205 patients with reactive stroma grade 1 and 2 vs. 16 patients with reactive stroma grade 0 and 3 (only one patient with grade 0).

In prostate cancer, the reactive stroma shows myofibroblasts that coexpress smooth-muscle marker ( $\alpha$-smooth-muscle actin) and mesenchymal marker (vimentin) and loss of late-stage smooth-muscle differentiation markers (desmin, calponin) (18). Based on these histological features, the only other study dealing with the prognostic value of reactive stroma in prostate carcinoma is Tomas' et al. (18). The authors quantified histochemically and immunohistochemically the stromal reaction in radical prostatectomies. Patients with a higher vimentin or lower desmin expression had a shorter disease-free period and on multivariate analysis only vimentin expression was a significant predictor of biochemical recurrence. Further studies on needle prostatic biopsies may show utility of histochemistry and immunohistochemistry for the analysis of reactive stroma.

\section{CONCLUSIONS}

Increasing reactive stroma grade on needle prostatic biopsies is significantly associated with several clinicopathologic adverse findings. However, only grade 3 predicts time and risk to biochemical recurrence following radical prostatectomy on univariate analysis. On multivariate analysis we have not been able to show that reactive stroma grade 3 is an independent predictor of biochemical recurrence factoring in preoperative PSA and other pathologic findings on biopsy. An additional limitation of reactive stroma grade 3 as prognostic factor is the relatively rare occurrence of this finding (only 5.3\% on needle biopsies).

\section{ACKNOWLEDGMENTS}

PSA = prostate specific antigen;

$\mathrm{SD}=$ standard deviation;

$\mathrm{CI}=$ confidence interval;

NC $=$ number of needle biopsy cores with carcinoma;

$\% \mathrm{NC}=$ percent of needle biopsy cores with carcinoma;

$\mathrm{mmAC}=$ total length of cancer in $\mathrm{mm}$ in all cores; $\%$ mmAC = percent of total length of cancer in $\mathrm{mm}$ in all cores.

\section{CONFLICT OF INTEREST}

None declared.

\section{REFERENCES}

1. Rønnov-Jessen L, Petersen OW, Bissell MJ: Cellular changes involved in conversion of normal to malignant breast: importance of the stromal reaction. Physiol Rev. 1996; 76: 69-125. 
2. Martin M, Pujuguet P, Martin F: Role of stromal myofibroblasts infiltrating colon cancer in tumor invasion. Pathol Res Pract. 1996; 192: 712-7.

3. Dvorak HF: Tumors: wounds that do not heal. Similarities between tumor stroma generation and wound healing. N Engl J Med. 1986; 315: 1650-9.

4. Tuxhorn JA, Ayala GE, Smith MJ, Smith VC, Dang TD, Rowley DR: Reactive stroma in human prostate cancer: induction of myofibroblast phenotype and extracellular matrix remodeling. Clin Cancer Res. 2002; 8: 2912-23.

5. Cunha GR, Hayward SW, Wang YZ, Ricke WA: Role of the stromal microenvironment in carcinogenesis of the prostate. Int J Cancer. 2003; 107: 1-10.

6. Zhang $\mathrm{Y}$, Nojima S, Nakayama $\mathrm{H}$, Jin $\mathrm{Y}$, Enza $\mathrm{H}$ : Characteristics of normal stromal components and their correlation with cancer occurrence in human prostate. Oncol Rep. 2003; 10: 207-11.

7. Rowley DR: What might a stromal response mean to prostate cancer progression? Cancer Metastasis Rev. 1998-1999; 17: 411-9.

8. De Wever 0, Mareel M: Role of tissue stroma in cancer cell invasion. J Pathol. 2003; 200: 429-47.

9. Dakhova 0, Ozen M, Creighton CJ, Li R, Ayala G, Rowley D, et al.: Global gene expression analysis of reactive stroma in prostate cancer. Clin Cancer Res. 2009; 15: 3979-89.

10. Niu YN, Xia SJ: Stroma-epithelium crosstalk in prostate cancer. Asian J Androl. 2009; 11: 28-35.

11. Olumi AF, Grossfeld GD, Hayward SW, Carroll PR, TIsty TD, Cunha GR: Carcinoma-associated fibroblasts direct tumor progression of initiated human prostatic epithelium. Cancer Res. 1999; 59: 5002-11.

12. Hayward SW, Cunha GR, Dahiya R: Normal development and carcinogenesis of the prostate. A unifying hypothesis. Ann $\mathrm{N}$ Y Acad Sci. 1996; 784: 50-62.

13. Cunha GR, Hayward SW, Dahiya R, Foster BA: Smooth muscle-epithelial interactions in normal and neoplastic prostatic development. Acta Anat (Basel). 1996; 155: 63-72.

14. De Wever 0, Mareel M: Role of tissue stroma in cancer cell invasion. J Pathol. 2003; 200: 429-47.

15. Tlsty TD: Stromal cells can contribute oncogenic signals. Semin Cancer Biol. 2001; 11: 97-104.
16. Ayala G, Tuxhorn JA, Wheeler TM, Frolov A, Scardino PT, Ohori $\mathrm{M}$, et al.: Reactive stroma as a predictor of biochemicalfree recurrence in prostate cancer. Clin Cancer Res. 2003; 9: 4792-801.

17. Yanagisawa N, Li R, Rowley D, Liu H, Kadmon D, Miles BJ, et al.: Stromogenic prostatic carcinoma pattern (carcinomas with reactive stromal grade 3 ) in needle biopsies predicts biochemical recurrence-free survival in patients after radical prostatectomy. Hum Pathol. 2007; 38: 1611-20.

18. Tomas D, Spajić B, Milosević M, Demirović A, Marusić Z, Kruslin B: Intensity of stromal changes predicts biochemical recurrence-free survival in prostatic carcinoma. Scand J Urol Nephrol. 2010; 44: 284-90.

19. Epstein JI, Allsbrook WC Jr, Amin MB, Egevad LL; ISUP Grading Committee: The 2005 International Society of Urological Pathology (ISUP) Consensus Conference on Gleason Grading of Prostatic Carcinoma. Am J Surg Pathol. 2005; 29: 1228-42.

20. Billis A, Magna LA, Ferreira U: Correlation between tumor extent in radical prostatectomies and preoperative PSA, histological grade, surgical margins, and extraprostatic extension: application of a new practical method for tumor extent evaluation. Int Braz J Urol. 2003; 29: 113-9; discussion 120.

21. Cookson MS, Aus G, Burnett AL, Canby-Hagino ED, D'Amico $A V$, Dmochowski RR, et al.: Variation in the definition of biochemical recurrence in patients treated for localized prostate cancer: the American Urological Association Prostate Guidelines for Localized Prostate Cancer Update Panel report and recommendations for a standard in the reporting of surgical outcomes. J Urol. 2007; 177: 540-5.

Correspondence address: Dr. Athanase Billis

Dep. of Anatomic Pathology

School of Medical Sciences State University of Campinas (Unicamp) Rua Tessália Vieira de Camargo, 126 Campinas, SP, 3083-887, Brazil Fax: + 5519 3289-3897

E-mail: athanase@fcm.unicamp.br 